itracerebral capillary vessels, but it was difficult to distinguish the boundary ne between the tumor and the normal tissue. The capsule of the meningioma as seen like a normal internal dural surface. Tissue biopsy was done Ifely through the trocar tube with the biopsy forceps. The interior of the tteral ventricle wall was inspected and pulsating vessels of the ventricular rall could be observed clearly.

But according to our experiences, we cosider that differential diagnosis $\mathrm{f}$ brain tumor with the encephaloscope was difficult owing to the thinning $f$ the color of the tumor through it's magnification by ten times under irect view. Therefore, the encephaloscope may be used to make tissue iopsy and treatment of intracerebral tumors which cannot be totally removed, $y$ inlaying radioactive substance through the trocar tube and removing it fter a certain period of time.

\title{
61. A Statistic Studies of Pediatric Neurosurgery in our Clinic
}

\author{
Shinken Kuramoto, Yasuhiro Nakamura and Toshikazu Fukuda \\ 1 st Department of Surgery, Kurume University School of Medicine
}

The neurological function of infancy or child must be examined in etail because they have long life in the future. For the purpose of investigating ediatric neurosurgery in our clinic, a statistical studies was performed. 83 ases under fifteen years of age and downwards were treated by us in the ast five years; trauma 51, hydrocephalus and congenital malformation 11 , rain tumor 7 , cerebral palsy and epileptic disease 7 , and others.

In the traumatic child, depression fracture and open injuries of the rain showed high average in comparision with those of adult patients. In the cases of brain tumor, 4 cases were subtentorial and others were supraentorial. In the hydrocephalus including one with myelomeningocele, five ases underwent ventriculo-atrial shunt operation. In our experience of V-A aunt operations with Dr. Spitz, we discussed some fault of present V-A lunt tube which have been made in Japan.

\section{Infectious Diseases of the Brain in Infants and Research of the Development of its Hematogenous Infection}

\author{
S. H. HuANG and A. HaKuBA
}

The Yodogaga Christian Hospital, Osaka Japan

In past $3 \frac{1}{2}$ years, we found 13 cases of infectious disease of the brain 